\title{
MODEL OF GEOMEDIA CONTAINING DEFECTS: COLLECTIVE EFFECTS OF DEFECTS EVOLUTION DURING FORMATION OF POTENTIAL EARTHQUAKE FOCI
}

\author{
I. A. Panteleev, O. A. Plekhov, O. B. Naimark \\ Institute of Continuous Media Mechanics, Ural Branch of RAS, Perm, Russia
}

\begin{abstract}
This paper describes the statistical thermo-dynamical evolution of an ensemble of defects in the geomedium in the field of externally applied stresses. The authors introduce 'tensor structural' variables associated with two specific types of defects, fractures and localized shear faults (Fig. 1). Based on the procedure for averaging of the structural variables by statistical ensembles of defects, a self-consistency equation is developed; it determines the dependence of the macroscopic tensor of defects-induced strain on values of external stresses, the original pattern and interaction of defects. In the dimensionless case, the equation contains only the parameter of structural scaling, i.e. the ratio of specific structural scales, including the size of defects and an average distance between the defects.
\end{abstract}

The self-consistency equation yields three typical responds of the geomedium containing defects to the increasing external stress (Fig. 2). The responses are determined from values of the structural scaling parameter. The concept of nonequilibrium free energy for a medium containing defects, given similar to the Ginzburg-Landau decomposition, allowed to construct evolutionary equations for the introduced parameters of order (deformation due to defects, and the structural scaling parameter) and to explore their solutions (Fig. 3).

It is shown that the first response corresponds to stable quasi-plastic deformation of the geomedium, which occurs in regularly located areas characterized by the absence of collective orientation effects. Reducing the structural scaling parameter leads to the second response characterized by the occurrence of an area of meta-stability in the behavior of the medium containing defects, when, at a certain critical stress, $\sigma_{m}\left(p_{m}\right)$, the orientation transition takes place in the ensemble of interacting defects, which is accompanied by an abrupt increase of deformation (Fig. 2). Under the given observation/averaging scale, this transition is manifested by localized cataclastic deformation (i.e. a set of weak earthquakes), which migrates in space at a velocity several orders of magnitude lower than the speed of sound, as a 'slow' deformation wave (Fig. 3). Further reduction of the structural scaling parameter leads to degeneracy of the orientation meta-stability and formation of localized dissipative defect structures in the medium. Once the critical stress $\sigma_{c}\left(p_{c}\right)$ is reached, such structures develop in the blowup regime, i.e. the mode of avalanche-unstable growth of defects in the localized area that is shrinking eventually. At the scale of observation, this process is manifested as brittle fracturing that causes formation of a deformation zone, which size is proportional to the scale of observation, and corresponds to occurrence of a strong earthquake.

On the basis of the proposed model showing the behavior of the geomedium containing defects in the field of external stresses, it is possible to describe main ways of stress relaxation in the rock massives - brittle large-scale destruction and cataclastic deformation as consequences of the collective behavior of defects, which is determined by the structural scaling parameter.

Results of this study may prove useful for estimation of critical stresses and assessment of the geomedium status in seismically active regions and be viewed as model representations of the physical hypothesis about the uniform nature of development of discontinuities/defects in a wide range of spatial scales.

Key words: geomedia defects, earthquake focus, stress relaxation, collective effects, metastability region, blow-up regime, deformation waves.

Recommended by Yu.L. Rebetsky

Citation: Panteleev I.A., Plekhov O.A., Naimark O.B., 2013. Model of geomedia containing defects: collective effects of defects evolution during formation of potential earthquake foci. Geodynamics \& Tectonophysics 4 (1), 37-51. doi:10.5800/GT-2013-4-1-0090. 


\title{
МОДЕЛЬ ГЕОСРЕДЫ С ДЕФЕКТАМИ: КОЛЛЕКТИВНЫЕ ЭФФЕКТЫ РАЗВИТИЯ НЕСПЛОШНОСТЕЙ ПРИ ФОРМИРОВАНИИ ПОТЕНЦИАЛЬНЫХ ОЧАГОВ ЗЕМЛЕТРЯСЕНИЙ
}

\author{
И. А. Пантелеев, О. А. Плехов, О. Б. Наймарк \\ Институт механики сплошных сред УрО РАН, Пермь, Россия
}

\begin{abstract}
Аннотация: В работе описана статистико-термодинамическая эволюция ансамбля дефектов в геосреде в поле внешнего приложенного напряжения. Авторами вводятся тензорные структурные переменные, ассоциированные с двумя характерными типами дефектов: трещинами и локализованными сдвигами (рис. 1). Процедура осреднения структурных переменных по статистическому ансамблю дефектов позволила получить уравнение самосогласования, определяющее зависимость макроскопического тензора деформации, индуцированной дефектами, от величины внешних напряжений, исходной структуры и взаимодействия дефектов, которое в безразмерном случае содержит только один параметр - параметр структурного скейлинга. Параметр структурного скейлинга определяется отношением характерных структурных масштабов: размером дефектов и средним расстоянием между дефектами.

В результате решения уравнения самосогласования получено три характерных реакции геосреды с дефектами на рост внешнего напряжения (рис. 2), которые определяются величиной параметра структурного скейлинга. Формулировка неравновесной свободной энергии для среды с дефектами в форме, аналогичной разложению ГинзбургаЛандау, позволила записать эволюционные уравнения для введенных параметров порядка (деформации, обусловленной дефектами, и параметра структурного скейлинга) и исследовать их собственные решения (рис. 3).

Показано, что первая реакция соответствует устойчивому квазипластическому деформированию среды, локализованному в регулярно расположенных пространственных областях, характеризующихся отсутствием коллективных ориентационных эффектов. Уменьшение параметра структурного скейлинга приводит ко второй реакции, которая характеризуется появлением области метастабильности в поведении среды с дефектами, когда при некотором критическом напряжении $\sigma_{m}\left(p_{m}\right)$ происходит ориентационный переход в ансамбле взаимодействующих дефектов, сопровождающийся резким скачком деформации (рис. 2). При этом на масштабе наблюдения (осреднения) этот переход проявляется в виде локализованной катакластической деформации (множества слабых землетрясений), мигрирующей по пространству со скоростью, на порядки меньшей скорости звука - «медленной» деформационной волны (рис. 3). Дальнейшее уменьшение параметра структурного скейлинга приводит к вырождению ориентационной метастабильности и формированию в среде локализованных диссипативных дефектных структур, которые при достижении критического напряжения $\sigma_{c}\left(p_{c}\right)$ развиваются в режиме с обострением - режиме лавинно-неустойчивого роста дефектов в локализованной пространственной области, уменьшающейся с течением времени. На масштабе наблюдения этот процесс проявляется в виде хрупкого разрушения с формированием зоны разрушения, соизмеримой с самим масштабом наблюдения, и соответствует появлению сильного землетрясения.

Таким образом, построенная модель поведения геосреды с дефектами в поле внешних напряжений позволяет описать основные способы релаксации напряжений массивами горных пород: хрупкое крупномасштабное разрушение и катакластическое деформирование, которые являются следствиями коллективного поведения дефектов, определяемого величиной параметра структурного скейлинга.

Полученные результаты могут быть полезны для оценки критических напряжений и состояний геосреды в сейсмоактивных районах, а также могут рассматриваться как модельные представления физической гипотезы о единстве природы развития несплошностей (дефектов) на широком спектре пространственных масштабов.
\end{abstract}

Ключевые слова: дефекты геосреды, очаг землетрясения, релаксация напряжений, коллективные эффекты, область метастабильности, режим с обострением, деформационные волны.

\section{1. ВВЕДЕНИЕ}

Современные данные свидетельствуют, что землетрясения обнаруживают признаки сложных динамических многоуровневых систем, включая пространственно-временную локализацию событий, автомодельность, миграцию активности по системам нарушений земной коры [Geilikman, Pisarenko, 1989; Sadovsky, 1989; Sadovsky et al., 1987; Sadovsky, Pisarenko, 1989; Hirata et al., 1987; Hirata, 1989]. Широкое распространение получили концепции, согласно которым разви- тие событий, предшествующих возникновению сильного землетрясения, может быть описано как поведение неравновесных критических систем [Sadovsky, Pisarenko, 1989; Tyupkin, 2004a, 2004b, 2004c; Knopoff, 1993; Sornette, 2000; Kossobokov et al., 2000]. Это означает, что с приближением момента сильного землетрясения характеристики индивидуального поведения структурных элементов геосреды становятся менее существенными, в отличие от коллективных эффектов, охватывающих все пространственно-временные масштабы системы и преобладающих в области возникно- 
вения наступающего землетрясения. Последние исследования основаны на гипотезе о том, что область, в которой наблюдается коррелированное появление сейсмических событий, изменяется со временем и может расти во время подготовки основного удара [Myachkin et al., 1975; Zaliapin et al., 2003; Tyupkin, di Giovambattista, 2005]. Под структурными элементами при этом понимаются дефекты геосреды того или иного масштабного уровня - поверхности механического нарушения сплошности, образующиеся в геосреде как в деформируемом твердом теле под действием внешних сил (трещины, разломы, границы блоков и т.д.). Наличие пространственно-иерархической структуры дефектов литосферы определяет существенную неоднородность полей напряжения того или иного масштаба, а как следствие влияет и на способ диссипации накопленной горными массивами упругой энергии (хрупкое крупномасштабное разрушение, катакластическое течение или локализованное пластическое течение) [Rebetskiy, 2007a]. Определяющая роль дефектов литосферы как физических носителей механизмов неупругого деформирования и разрушения, реализуемых на всем спектре пространственных масштабов, является основным положением нового научного направления - физической мезомеханики [Panin, 1998; Panin et al., 2004]. Впервые на аналогию особенностей процесса разрушения и явлений, которые наблюдаются в сейсмически активных зонах, обращено внимание в работах С.В. Гольдина [Goldin, 2002, 2004].

Наиболее известной и общепризнанной моделью подготовки очага землетрясения, рассматривающей эволюцию дефектов как главный механизм формирования очага, является модель лавинно-неустойчивого трещинообразования (ЛНТ-модель) [Myachkin, 1978]. Основой модели послужили результаты лабораторных экспериментов по разрушению горных пород с одновременной регистрацией акустической эмиссии [Lockner et al., 1992; Sobolev, Ponomarev, 2003]. Согласно ЛНТ-модели, процесс формирования источника землетрясения состоит из трех стадий. На первой стадии под влиянием медленного увеличения тектонических напряжений происходит однородное накопление трещин в объеме $\Omega$. После того как плотность трещин $v$ достигает критической величины в относительно малой области $\Omega_{S}<\Omega$, состояние среды входит во вторую стадию, которая характеризуется заметным увеличением трещинообразования в $\Omega_{S}$ и ростом области $\Omega_{S}$. Эта стадия связана с образованием потенциального источника землетрясения, а область $\Omega_{S}(t)$ - с образованием потенциального региона источника. Общая скорость деформации в области $\Omega_{S}(t)$ увеличивается в результате вклада деформации, вызванной перемещениями вдоль берегов трещин. Основная физическая особенность потенциальной области источника заключается в том, что при выполнении условия $v>v_{c r}{ }^{(1)}$ взаимодействие трещин увеличивает вероятность растрескивания материала и приводит к самоорганизации процесса разрушения. Третью стадию отличает начало неустойчивой деформации, локализованной в узкой области развивающегося макроразрыва. Эта стадия начинается после того, как плотность трещин достигает второй критической точки $v_{c r}{ }^{(2)}$ и развивается, как правило, благодаря упругой энергии, накопленной в среде. Существенный момент ЛНТ-модели - иерархия трещин, при которой переход от одной стадии к другой сопровождается укрупнением масштаба и эволюцией дефектов. При укрупнении трещин не требуется напряжений, близких к предельным.

Одним из первых формализованных подходов для описания указанных стадий является реологическая модель В.П. Мясникова и В.А. Ляховского [Lyakhovsky, Myasnikov, 1985; Lyakhovsky et al., 1997], учитывающая влияние упругой деформации, вязкой релаксации и развития повреждений в материале. Согласно этой модели плотность свободной энергии может быть записана в виде:

$$
F_{e}=F_{0}+\frac{1}{\rho}\left(\frac{\lambda(\alpha)}{2} I_{1}^{2}+\mu(\alpha) I_{2}-\gamma(\alpha) I_{1} \sqrt{I_{2}}\right),
$$

где $I_{1}=\sum_{i} \varepsilon_{i i}, I_{2}=\sum_{i j} \varepsilon_{i j} \varepsilon_{i j}-$ первый и второй инварианты тензора деформации, $\rho$ - плотность материала, $\quad \lambda(\alpha)=\lambda_{0}-\alpha \lambda_{r}, \quad \mu(\alpha)=\mu_{0}-\alpha \mu_{r}, \quad \gamma(\alpha)=\alpha \gamma_{r}$, $\lambda_{0}, \mu_{0}-$ константы Ляме неповрежденного материала, $\lambda_{r}, \mu_{r}, \gamma_{r}-$ параметры, характеризующие вклад повреждений в упругие свойства, $F_{0}=F_{0}(T)$ - константа в изотермическом случае. Безразмерная переменная $\alpha(0<\alpha \leq 1)$ определяет степень поврежденности материала. Выражение для плотности свободной энергии получено в предположении об отсутствии взаимодействия между трещинами. В рамках данной модели может быть описана первая стадия развития формирования источника землетрясения. Для описания перехода от диффузного накопления трещин к состоянию самоорганизованного растрескивания в работе [Lyakhovsky et al., 1997] вводится дополнительная переменная $\varphi \geq 0$, определяющая степень взаимодействия между разрывами. По аналогии с фазовыми переходами параметр порядка $\alpha$ имеет смысл обратной температуры. В этом случае плотность свободной энергии с учетом степени взаимодействия между разрывами может быть представлена в виде:

$$
\begin{aligned}
& F\left(\varepsilon_{i j}, \alpha, \varphi, \nabla \alpha, \nabla \varphi\right)= \\
& =F_{e}\left(\varepsilon_{i j}, \alpha\right)+F_{i}\left(\varepsilon_{i j}, \alpha, \varphi, \nabla \alpha, \nabla \varphi\right),
\end{aligned}
$$

где $F_{e}\left(\varepsilon_{i j}, \alpha\right)$ задается соотношением (1). При этом кинетическое уравнение для параметра поврежденности 
$\alpha$ представляется в виде уравнения Гинзбурга-Ландау:

$$
\frac{d \alpha}{d t}=\left\{\begin{array}{c}
-K_{\alpha} \frac{\partial F(\varepsilon, \alpha, \varphi)}{\partial \alpha}, \frac{\partial F(\varepsilon, \alpha, \varphi)}{\partial \alpha} \leq 0 \\
0, \frac{\partial F(\varepsilon, \alpha, \varphi)}{\partial \alpha}>0
\end{array} .\right.
$$

В случае учета пространственной неоднородности параметра поврежденности в выражении для компонент тензора напряжения появляется слагаемое, отражающее эффекты нелокальности в терминах параметра $\varphi$.

Данный подход позволил описать такие эффекты, как ускорение сейсмического энерговыделения [Varnes, 1989; Bowman et al., 1998; Sobolev, Tyupkin, 2000] и рост корреляционной длины слабой сейсмичности перед большим землетрясением [Rundle et al., 1999; Zöller et al., 2001; Tyupkin, di Giovambattista, 2005]. В квазистатическом приближении он позволяет описать две из трех стадий подготовки источника землетрясения.

Попытка построения модели развития сейсмических событий с позиций статистической физики была предпринята в [Rundle, 1988, 1989; Rundle et al., 1997] при рассмотрении системы локализованных сдвигов $s(x, t)$ по нарушениям в земной коре с заданной реологией, подвергнутой нагружению некоторым полем напряжений. Статическое равновесие такой системы определяется соотношением:

$$
\sigma_{\mathrm{e}}\left[\mathrm{x}, \mathrm{t}, \mathrm{s}\left(\mathrm{x}^{\prime}, \mathrm{t}^{\prime}\right), \mathrm{p}\right]=\sigma_{\mathrm{f}}[\mathrm{x}, \mathrm{t}, \mathrm{s}(\mathrm{x}, \mathrm{t})],
$$

где $\sigma_{\mathrm{e}}(\mathrm{x}, \mathrm{t})$ - упругие напряжения, определяемые смещением основания исходного нарушения земной коры, а также напряжениями, возникающими от сдвигов на нарушениях с координатами $\left(\mathrm{x}^{\prime}, \mathrm{t}^{\prime}\right) ; \sigma_{\mathrm{f}}(\mathrm{x}, \mathrm{t}),-$ напряжения трения на нарушениях земной коры, ассоциированные со сдвигами s(x,t). Полученная модель позволила установить переходы от состояний системы с глобальным минимумом энергии к метастабильному состоянию при вариации безразмерного параметра. Последний определяет баланс соотношения энергий упругих возмущений в окрестности области сдвига, локализованного на нарушении земной коры, и длинно-коррелированных возмущений «эффективного сдвигового поля», инициирующего, наряду с упругой составляющей поля, локальный сдвиг на выделенном нарушении.

Существенным недостатком современных моделей подготовки очага землетрясения является отсутствие формализованного описания с единых позиций каждой из трех стадий как этапов эволюции сплошной среды с дефектами под действием внешних приложенных сил. Отдельная нерешенная проблема заключается в строгом математическом описании перехода и кинетики развития среды на третьей стадии подготовки землетрясения, при которой происходит неустойчивый катастрофический рост поврежденности в локализованной пространственной области, уменьшающейся с течением времени. Характерное время протекания этого процесса может быть на порядки меньше характерного времени изменения внешней нагрузки. В работе [Туирkin, 2007] подчеркивается, что для описания заключительной стадии необходимо использовать методологию, развитую в [Naimark, 1998], и рассматривать динамику изменения напряженно-деформированного состояния и роста поврежденности в геосреде с образованием пространственно-временных локализованных структур.

Цель настоящей работы - построение модели подготовки потенциального очага землетрясения как результата эволюции дефектов геосреды на различных масштабных уровнях. В ее основе лежит статистикотермодинамическая модель среды с дефектами, учитывающая коллективные эффекты в ансамбле типичных дефектов - локальных сдвигов и трещин. При этом предполагается, так же как и в ЛНТ-модели, что источник землетрясения не связан с существующим разломом, а формируется благодаря коллективному поведению ансамбля дефектов. Особенности коллективного взаимодействия описываются с использованием двух независимых термодинамических переменных упругопластической среды с дефектами: тензора плотности дефектов, имеющего смысл деформации, обусловленной дефектами, и параметра структурного скейлинга, характеризующего роль масштабных эффектов и взаимодействие дефектов на различных масштабных уровнях.

В работе [Naimark, 2008] показано, что реакция материала с дефектами на внешнюю нагрузку включает стадию лавинообразного роста плотности дефектов в локальной области, уменьшающейся с течением времени. Такой сценарий развития дефектов в материале совпадает с распространенным качественным описанием третьей стадии подготовки землетрясения согласно ЛНТ-модели и позволяет использовать данный подход для моделирования кинетики плотности дефектов на завершающей стадии подготовки.

\section{2. СТАТИСТИЧЕСКАЯ МОДЕЛЬ ПОВЕДЕНИЯ МАТЕРИАЛОВ С ДЕФЕКТАМИ}

Использование методов статистической физики для описания поведения произвольного ансамбля элементов априори подразумевает рассмотрение двух масштабов $-L_{a}$ и $L_{a-1}$, отличающихся как минимум на порядок величины. При этом масштаб $L_{a-1}$ соотносится с характерными размерами отдельного элемента, а масштаб $L_{a}$ соответствует характерным размерам всего ансамбля, поведение которого на этом масштабе 

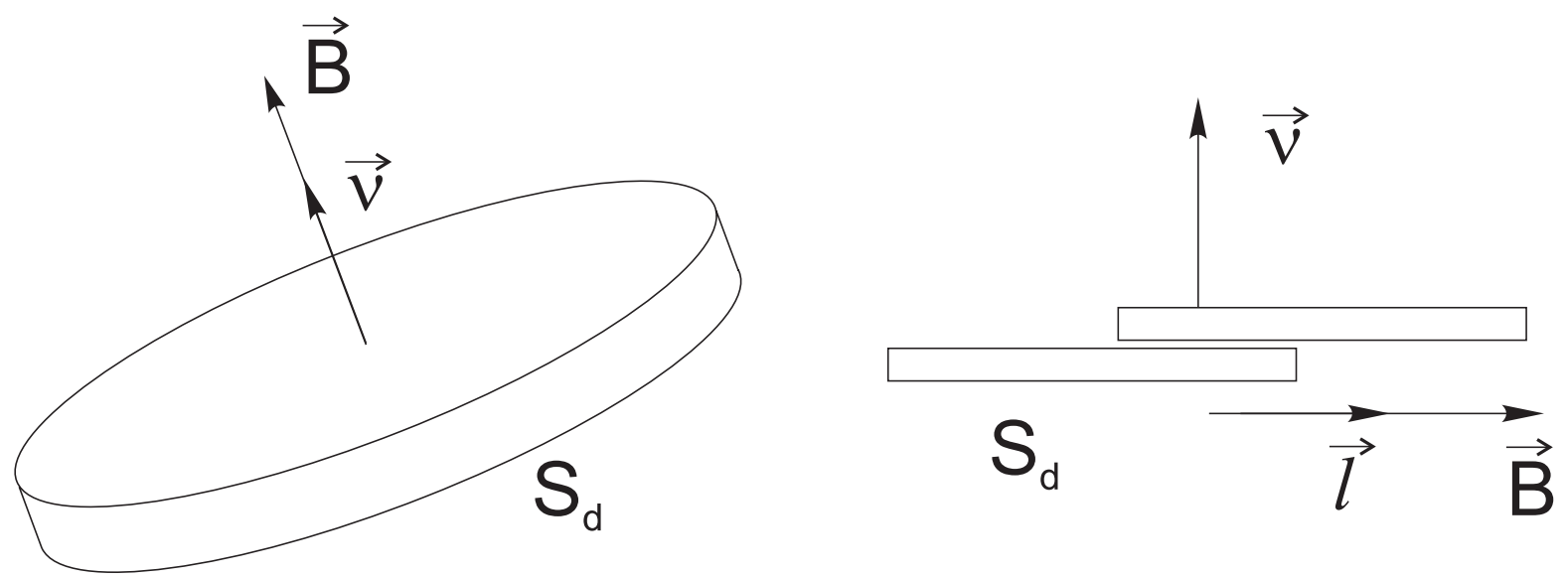

Рис. 1. Характерные типы дефектов: дискообразная трещина и локализованный сдвиг ( $\vec{v}-$ единичный вектор нормали к основанию площадки сдвига или основанию микротрещины, $\vec{l}$ - единичный вектор в направлении сдвига).

Fig. 1. Typical types of defects: disc-shaped fracture and localized shear fault ( $\vec{v}$ - unit vector normal to the base of the shear fault or the base of the micro-fracture; $\vec{l}$ - unit vector in the direction of the shear faulr).

можно описать полевыми уравнениями для некоторых осредненных величин. При описании тектонофизических процессов выбор масштаба осреднения играет важную роль и зависит от того, на каком масштабе планируется проводить анализ напряженно-деформированного состояния среды. Согласно [Rebetskiy, $2007 b]$, для этих процессов могут быть выделены сублокальный, локальный, субрегиональный и региональный масштабы осреднения, отличающиеся друг от друга на порядок величины. Процедура построения модели подготовки потенциального очага землетрясения, представленная ниже, не зависит от выбора конкретной пары масштабов, поэтому, не нарушая общности, будем рассматривать произвольную пару соседних масштабов - $L_{a-1}$ и $L_{a}$, которые в дальнейшем для краткости будем называть микро- и макромасштабами соответственно.

Локализованные сдвиги и трещины, определяющие структурные изменения и осуществляющие перенос импульса в твердых телах, могут быть введены в рассмотрение в качестве дополнительных независимых переменных системы, отражающих роль структурных изменений в термодинамических, статистических и динамических свойствах рассматриваемых сред. Введем структурные переменные, ассоциированные с микротрещинами и микросдвигами, как аналоги тензоров дислокационной плотности (согласно [Naimark, 1982]). Эти дефекты описываются симметричными тензорами вида $s_{i k}=1 / 2 s\left(v_{i} l_{k}+l_{i} v_{k}\right)$ для случая микросдвигов и $s_{i k}=s v_{i} v_{k}-$ для дискообразных трещин (рис. 1). Здесь $s=S_{d}|B|-$ интенсивность сдвига на площадке $\vec{S}_{d}=S_{d} \vec{v}$ или объем микротрещины, $\vec{v}-$ единичный вектор нормали к основанию площадки сдвига или основанию микротрещины, $\vec{l}$ - единичный вектор в направлении сдвига.

Кинетические уравнения, описывающие временную эволюцию введенных структурных переменных, должны отражать влияние двух действующих факторов $[M a, 1980]:$ регулярное движение и действие флуктуаций.

В общем случае кинетические уравнения, описывающие эволюцию единичного дефекта, могут быть записаны в форме уравнения Ланжевена для эволюции случайной величины:

$$
\dot{s}_{i k}=K_{i k}\left(s_{l m}\right)-F_{i k}(t),
$$

где $K_{i k}, F_{i k}$ - регулярная и стохастическая части действующих на дефект сил. Для получения информации о распределении макроскопических величин перейдем к эквивалентной форме записи случайного процесса в виде уравнения Фоккера-Планка для функции распределения $W\left(s_{i k}, t\right)$, предполагая, что корреляции между значениями случайной силы в различные моменты времени отсутствуют, а среднее значение в любой момент времени равно нулю:

$$
\frac{\partial}{\partial t} W=-\frac{\partial}{\partial s_{i k}} K_{i k} W+\frac{1}{2} Q \frac{\partial^{2}}{\partial s_{i k} \partial s_{i k}} W .
$$

В ряде работ [Barenblatt, Botvina, 1982; Botvina, Barenblatt, 1985] при описании процесса накопления повреждений используется предположение об автомодельности распределения дефектов на различных стадиях процесса разрушения. Статистическое самоподобие распределения дефектов отражает важный факт, заключающийся в том, что функция распределения 
имеет постоянную форму для различных моментов времени и соответствует стационарному решению уравнения Фоккера-Планка с параметрической зависимостью распределения от некоторого параметра подобия.

Анализ экспериментальных данных и полевых наблюдений показал, что подобие режимов накопления повреждений может наблюдаться в различных процессах деформирования твердых тел на пространственных масштабах от микрон до сотен и тысяч километров [Botvina et al., 1995; Sherman, 1977; Leonov, 2011]. Kaчественная интерпретация гипотезы подобия предполагает, что геометрическое распределение дефектов на заключительной стадии деформирования представляет увеличенную копию этого распределения на более ранней стадии. Таким образом, подобие позволяет ввести в рассмотрение средний размер дефекта и его характерную энергию для того, чтобы установить их соответствие приложенной нагрузке. С учетом гипотезы статистического подобия, решение уравнения (6) имеет вид [Naimark, 1982]:

$$
W=Z^{-1} \operatorname{Exp}\left(-E\left(s_{i k}\right) / Q\right),
$$

где $Z$ - нормирующий множитель, $Q$ - потенциал, совпадающий по смыслу с усредненной энергией, приходящейся на характерные степени свободы в системе дефектов, $E\left(s_{i k}\right)$ - энергия единичного дефекта.

Макроскопическая деформация $p_{i k}$, вызванная дефектами, определяется осреднением $s_{i k}$ по статистическому ансамблю дефектов соответствующего типа, микротрещинам и микросдвигам: $p_{i k}=n\left\langle s_{i k}\right\rangle$, где $n-$ концентрация дефектов. В работе [Naimark, 2004] показано, что изменение энергии системы, связанной с формированием локализованных мод деформации, обусловленной дефектами типа микросдвигов и микротрещин, может быть записано в форме $E-E_{0}=-H_{i k} s_{i k}+\alpha s_{i k}{ }^{2}$ и включает член $H_{i k} s_{i k}$, который отражает взаимодействие дефектов с внешним (эффективным) полем, а также взаимодействие между дефектами. «Эффективное поле» $H_{i k}$ может быть представлено в виде суммы внешнего поля и «среднего» поля, индуцированного дефектами: $H_{i k}=\gamma \sigma_{i k}+\lambda p_{i k}$, где $\sigma_{i k}-$ макроскопический тензор напряжений, $p_{i k}=n\left\langle s_{i k}\right\rangle-$ макроскопический тензор плотности дефектов, $n-$ концентрация дефектов, $\lambda$ и $\gamma$ - параметры материала. Квадратичный член $\alpha s_{i k}{ }^{2}$ отражает флуктуацию энергии поля дисторсии, возникающей в окрестности зародыша дефекта (существующего нарушения земной коры) под действием «эффективного поля» и флуктуаций «шума».

С учетом вида функции распределения и изменения энергии одиночного дефекта макроскопическое значе- ние тензора плотности дефектов $p_{i k}$ (деформации, обусловленной дефектами) может быть записано в виде:

$$
p_{i k}=n \int s_{i k} W(s, v, l) d s d^{3} v d^{3} l .
$$

При этом выбор типа микроскопического структурного параметра $s_{i k}$ позволяет получить уравнение для шаровой части (в случае $s_{i k}=s v_{i} v_{k}$ ) и девиаторной части тензора деформации, индуцированного дефектами (в случае $s_{i k}=1 / 2 s\left(v_{i} l_{k}+l_{i} v_{k}\right)$ ), которое описывает трещинную дилатансию и изменение формы среды за счет множественных локальных сдвигов соответственHo.

Переход к безразмерным переменным

$$
p_{i k}=1 / n \sqrt{\alpha / Q} p_{i k}, s_{i k}=\sqrt{\alpha / Q} s_{i k}, \sigma_{i k}=\sigma_{i k} / \sqrt{Q \alpha}
$$

позволяет представить соотношение (8) в виде уравнения самосогласования:

$$
p_{i k}=\int s_{i k} Z^{-1} \exp \left(\left(\sigma_{i k}+\frac{1}{\delta} p_{i k}\right) s_{i k}-s_{i k}^{2}\right) d s_{i k},
$$

которое включает единственный безразмерный параметр $\delta=\alpha / \lambda n$.

Размерностный анализ величин, входящих в (10), позволяет получить оценку

$$
\alpha \sim \frac{G}{V_{0}}, \quad \lambda \sim G, \quad n \sim R^{-3}
$$

где $G$ - эффективная характеристика «упругости» среды, $V_{0} \sim r_{0}^{3}$ - характерный объем зародыша, $R$ среднее расстояние между дефектами.

В результате получаем, что данный параметр представляет отношение двух характерных масштабов в среде с дефектами

$$
\delta \sim\left(R / r_{0}\right)^{3}
$$

где $r_{0}$ - характерный размер зародышей дефектов, $R$ - расстояние между дефектами, которое отражает «статистическую автомодельность» в поведении дефектов различных структурных уровней. Дополнительный параметр порядка $\delta$ следует естественным образом из решения статистической задачи и имеет структуру, аналогичную концентрационному параметру, введенному в работах [Zhurkov, 1968; Kuksenko, 1986].

Решение уравнения (10) для одномерного случая позволило получить три характерных нелинейных зависимости компонент шаровой $p^{0}$ и девиаторной 


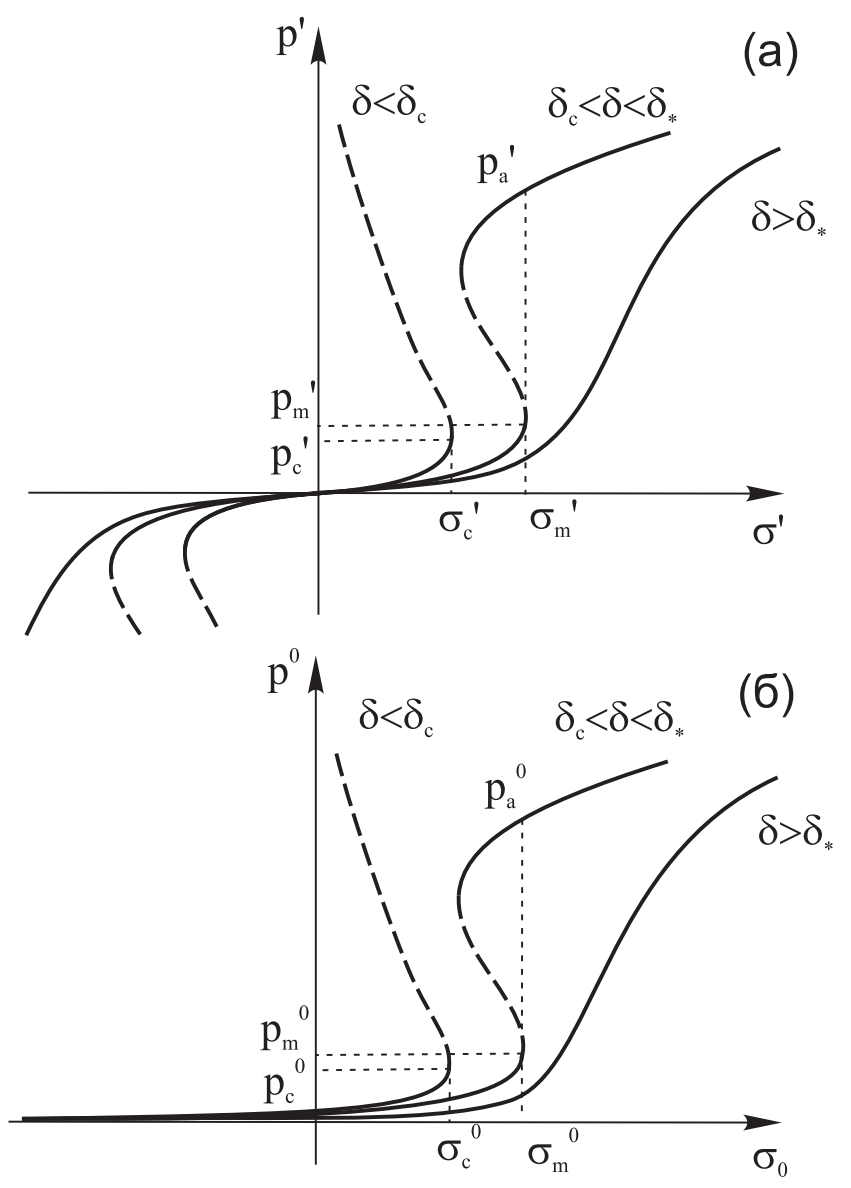

Рис. 2. Характерные зависимости девиаторной (a) и шаровой (б) частей деформации, обусловленной дефектами, от приложенного напряжения для различных значений параметра структурного скейлинга.

Fig. 2. Typical relationships between deviatoric (a)/ spherical (b) parts of deformation, that occurs due to defects caused by the applied stress, and different values of the structural scaling parameter.

частей $p^{\prime}$ тензора деформации, обусловленной дефектами от приложенного напряжения для различных значений параметра структурного скейлинга $\delta$ $\left(\delta>\delta_{*} \approx 1.3 ; \quad \delta_{\text {с }}<\delta<\delta_{*} ; \quad \delta<\delta_{\text {с }} \approx 1\right.$, где $\delta_{\text {с }}, \delta_{*}-$ точки бифуркации решения) (рис. 2).

При этом полученные зависимости для шаровой и девиаторной частей деформации, обусловленной дефектами, совпадают только в случае приложенных растягивающих напряжений. Различие в случае сжимающих приложенных напряжений объясняется типом выбранных дефектов, описываемых соответствующим структурным параметром $s_{i k}$.

Статистический подход к описанию поведения среды с дефектами под внешней нагрузкой дал возможность записать неравновесный потенциал, который описывает развитие локализованных дисторсий при различных сценариях, связанных с типами нелинейности самого потенциала, и представляет «минимальное разложение» для неравновесной свободной энергии.
Потенциал задается полиномом 6-й степени и имеет форму, подобную форме разложения Гинзбурга-Ландау:

$$
\begin{aligned}
& F=\frac{1}{2} A\left(\delta, \delta_{*}\right) p_{i k}^{2}-\frac{1}{4} B p_{i k}^{4}+ \\
& +\frac{1}{6} C\left(\delta, \delta_{c}\right) p_{i k}^{6}-D \sigma_{i k} p_{i k}+\chi\left(\nabla p_{i k}\right)^{2} .
\end{aligned}
$$

Градиентный член в выражении (12) отвечает за эффекты нелокальности в ансамбле взаимодействующих дефектов; $A, B, C, D$ и $\chi$ - параметры, характеризующие нелинейные и нелокальные свойства континуума с взаимодействующими дефектами. Например, в случае чистого сдвига (обозначим $\sigma=\sigma_{x z}, p=p_{x z}$ ) кинетика параметров порядка задается известным эволюционным неравенством $\partial F / \partial t=\dot{p} \partial F / \partial p+\dot{\delta} \partial F /$ $/ \partial \delta<0$, откуда

$$
\begin{aligned}
& \frac{d p}{d t}=-A_{p}\left[\begin{array}{l}
A\left(\delta, \delta_{*}\right) p-B p^{3}+C\left(\delta, \delta_{c}\right) p^{5}- \\
-D \sigma-\nabla_{i}\left(\chi \nabla_{l} p\right)
\end{array}\right], \\
& \frac{d \delta}{d t}=-A_{\delta}\left(\frac{1}{2} \frac{\partial A}{\partial \delta} p^{2}-\frac{1}{6} \frac{\partial C}{\partial \delta} p^{6}\right),
\end{aligned}
$$

где $A_{p}$ и $A_{\delta}$ - кинетические коэффициенты.

Как следует из решения уравнения (10), переходы через точки бифуркации $\delta_{c}$ и $\delta_{*}$ приводят к резкому изменению типов коллективных мод для параметра порядка $p$. Сценарии переходов определяются групповыми свойствами уравнения (13), которые различны для разных интервалов значений параметра структурного скейлинга $\delta$.

Для достаточно больших $\delta$ (когда размеры дефектов намного меньше характерного расстояния между ними) собственное решение кинетического уравнения для деформации, обусловленной дефектами, имеет вид периодических структур с пространственным периодом $\Lambda$ (рис. 3). Зависимость $p(\sigma)$ является монотонной (см. рис. 2), а свободная энергия $F(p, \delta)$ имеет один минимум, определяющий равновесную при данной нагрузке плотность дефектов.

Физически это описывает ситуацию, когда для теоретически любых растягивающих напряжений существует равновесная концентрация дефектов. В диапазоне $\delta_{\text {с }}<\delta<\delta_{*}$ наблюдается метастабильность по параметру $p$, связанная с ориентационными степенями свободы дефектов. При $\delta \rightarrow \delta_{*}$ периодическое собственное решение трансформируется в решение автосолитонного типа $p(\zeta)=p(x-V t)$ (рис. 3). Амплитуда волны, ее ширина и скорость волнового фронта определяются 


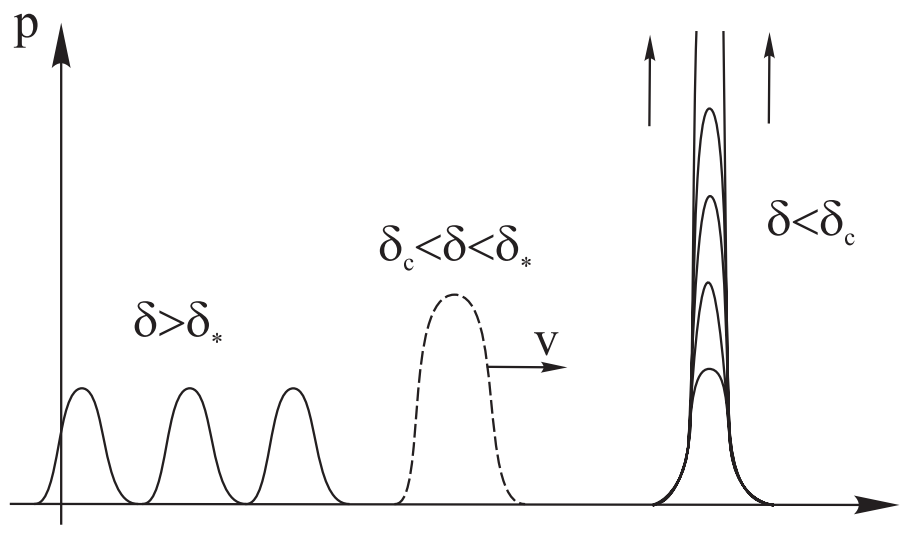

$\mathrm{X}$

Рис. 3. Автомодельные решения кинетического уравнения для деформации, обусловленной дефектами: периодические пространственные структуры (при $\delta>\delta_{*}$ ), автосолитонные волны (при $\delta_{\text {с }}<\delta<\delta_{*}$ ) и локализованные диссипативные структуры обострения $\left(\delta<\delta_{C}\right)$.

Fig. 3. Automodeling solutions of the kinetic equation for deformation due to defects: periodic spatial structures $\left(\delta>\delta_{*}\right)$; autosoliton waves $\left(\delta_{\mathrm{c}}<\delta<\delta_{*}\right.$ ), and localized dissipative structures of peaking $\left(\delta<\delta_{c}\right)$.

параметрами неравновесного ориентационного перехода:

$$
\begin{aligned}
& p=\frac{1}{2} p_{\alpha}\left[1-\operatorname{th}\left(\zeta l^{-1}\right)\right], \quad l=\frac{4}{p_{\alpha}}\left(2 \frac{\chi}{A}\right)^{1 / 2}, \\
& V=\chi A\left(p_{\alpha}-p_{m}\right) / 2 \zeta^{2},
\end{aligned}
$$

где $\left(p_{\alpha}-p_{m}\right)$ - скачок величины $p$ в ходе ориентационного перехода. Зависимость свободной энергии от деформации, обусловленной дефектами, имеет два минимума, разделенных потенциальным барьером, переход через который происходит при достижении критического напряжения $\sigma_{m}$ и сопровождается скачкообразным ростом плотности дефектов.

Переход через точку бифуркации $\delta_{c}$ сопровождается формированием в ансамбле дефектов рассматриваемого пространственного масштаба коллективных мод нового типа - диссипативных структур обострения пространственно-временных структур, характеризующихся взрывообразной кинетикой зарождения и роста дефектов (рис. 3). При этом равновесное накопление дефектов в таком случае возможно только до некоторого критического значения $\sigma_{c}\left(p_{c}\right)$, при переходе через которое начинается лавинообразный рост дефектов (формирование очага макроскопического хрупкого разрушения) на некотором пространственном масшта- бе. В работе [Panteleev et al., 2011] показано, что автомодельные решения уравнения (13) соответствуют набору автомодельных решений квазилинейного параболического уравнения LS-типа (по классификации С.П. Курдюмова [Kurdyumov, Malinetsky, 1983]), согласно которому лавинообразный рост дефектов происходит на пространственном масштабе, уменьшающемся с течением времени. Такой сценарий развития дефектов совпадает с распространенным качественным описанием третьей стадии подготовки землетрясения в соответствии с ЛНТ-моделью, что позволяет использовать данный подход для моделирования эволюции дефектов на завершающей стадии подготовки с позиций механики деформируемого твердого тела с дефектами [Panteleev et al., 2012].

\section{3. ОБСУЖДЕНИЕ РЕЗУЛЬТАТОВ}

Формулировка задачи статистической физики для описания эволюции ансамбля дефектов в поле внешнего приложенного напряжения позволила естественным образом ввести единственный безразмерный параметр, определяемый отношением двух характерных масштабов: среднего размера дефектов и среднего расстояния между ними. Данный параметр является единственным параметром, определяющим особенности поведения геосреды с дефектами в процессе деформирования и разрушения, его структура подтверждает высказанную гипотезу о «статистической автомодельности». В результате можно утверждать, что разрабатываемая физическая теория согласуется с гипотезой о единстве природы развития несплошностей на широком спектре пространственных масштабов, высказанной во второй половине прошлого века М.В. Гзовским [Gzovsky, 1975] и многократно подтвержденной как в лабораторных экспериментах, так и в полевых наблюдениях [Sherman et al., 1999, 2000; Sherman, Seminsky, 2010]. Однако из подобия механизмов развития дефектов на различных пространственных масштабах не следует, что величины критических напряжений $\sigma_{c}\left(p_{c}\right)$, $\sigma_{m}\left(p_{m}\right)$, определяющих соответствующие ориентационные переходы в ансамблях дефектов, совпадают для каждого из них. Размер области метастабильности среды с дефектами на каждом из масштабов, а как следствие, и критические значения напряжений определяются уровнем напряжений и развитостью дефектов на масштабе на порядок меньше рассматриваемого.

Особенностью построенной модели поведения геосреды с дефектами в поле приложенных внешних напряжений (в отличие от двухстадийной модели разрушения В.С. Куксенко, С.Н. Журкова [Zhurkov, 1968; Kuksenko, 1986]) является то, что параметр структурного скейлинга, имеющий структуру, аналогичную концентрационному параметру, не является постоянным. Его величина определяет реализацию соответ- 
ствующего механизма деформирования среды с дефектами (а как следствие, и способ релаксации накопленных напряжений), а эволюция определяет смену этого механизма. При $\delta>\delta_{*}$ имеет место устойчивое квазипластическое деформирование среды, локализованное в регулярно расположенных пространственных областях, характеризующееся отсутствием коллективных ориентационных эффектов. Примерами таких областей, по мнению авторов, могут служить внутриплитные зоны концентрированной деформации [Leonov, 2011] и зоны регулярности в нарушенности разрывами субстрата разломных зон [Seminsky, 2009], активно исследуемые в настоящее время. Интервал $\delta_{\text {с }}<\delta<\delta_{*}$ значений параметра структурного скейлинга характеризуется появлением области метастабильности в поведении среды с дефектами, когда при некотором критическом напряжении $\sigma_{m}\left(p_{m}\right)$ происходит ориентационный переход в ансамбле взаимодействующих дефектов, сопровождающийся резким скачком деформации. При этом на избранном масштабе осреднения этот переход проявляется в виде локализованной истинной катакластической деформации [Rebetskiy, $2007 b]$, мигрирующей по пространству со скоростью, на порядки меньшей скорости звука, - «медленной» деформационной волны, являющейся неотъемлемым фундаментальным свойством самоорганизации деформируемой среды. В работах [Goldin et al., 2002; Makarov, 2005, 2008] подчеркивается особая роль «медленных движений» в процессах деструкции твердого тела (от лабораторного образца до масштаба литосферы) как одного из основных способов переноса энергии.

Дальнейшее уменьшение среднего расстояния между дефектами $\left(\delta<\delta_{c}\right)$ приводит к вырождению ориентационной метастабильности и формированию в среде локализованных диссипативных дефектных структур, которые при достижении критического напряжения $\sigma_{c}\left(p_{c}\right)$ развиваются в режиме с обострением - режиме лавинно-неустойчивого роста дефектов в локализованной пространственной области, уменьшающейся с течением времени. На масштабе наблюдения этот процесс проявляется в виде хрупкого разрушения с формированием зоны разрушения, соизмеримой с самим масштабом наблюдения. Таким образом, эволюция дефектной подсистемы в режиме с обострением является заключительной стадией перед переходом процесса деформирования и разрушения на следующий пространственный масштаб, что, согласно работам [Makarov et al., 2007; Makarov, 2008], является общим свойством всех деформируемых твердых тел и сред. Теоретические предположения о появлении обостряющихся локализованных диссипативных структур при локализации разрушения впервые были высказаны в работе [Naimark, 1982] и получили свое развитие в работах [Naimark et al., 2000; Naimark, Davydova, 1996;
Naimark, 2003, 2006; Makarov, 2008]. Фундаментальным свойством реализации режима с обострением [Kurdyumov, Malinetsky, 1983; Kurdyumov, 2006] является «замораживание» развития процесса деструкции на всем рассматриваемом пространственном масштабе кроме локальной области, на которой это обострение будет происходить. Физически это проявляется в виде зон затишья, которые имеют место перед землетрясениями [Goldin, 2004; Fedotov, 2005; Makarov, 2011]. Особое внимание хотелось бы обратить на важное следствие из полученных результатов: предельное напряжение, требуемое для начала формирования очага макроскопического хрупкого разрушения, меньше, нежели для реализации локализованного катакластического течения, - высокий уровень локальных напряжений не является индикатором и необходимым условием начала хрупкого разрушения. Этот вывод согласуется с последними результатами по тектонофизической реконструкции природных напряжений в ряде районов крупных землетрясений, выполненныой методом катакластического анализа разрывных смещений, активно развиваемого коллективом Ю.Л. Ребецкого [Rebetskiy, 2003, 2007a, 2009a, 2009b]. По итогам реконструкции обнаружено, что очаги крупных землетрясений не совпадают с областями максимального уровня напряжений, а располагаются в области среднего уровня девиаторных и изотропных напряжений, соседствующей с участком большого градиента этих напряжений.

К обособленным дискуссионным вопросам в рамках любой разрабатываемой модели формирования очага землетрясения относится вопрос использования ее положений, результатов для построения прогностических теорий. В работе [Strakhov, 1989], посвященной анализу проблем фундаментальной сейсмологии в области прогноза сейсмической опасности, указывается на необходимость создания новой парадигмы для решения проблем прогноза, базирующейся на знании физических закономерностей хрупкого разрушения, возникающего в больших массивах горных пород. В представленной авторами модели поведения геосреды с дефектами в поле приложенных внешних напряжений девиаторная часть структурного параметра $s_{i k}$, ассоциированная с локальным сдвигом, имеет структуру, аналогичную тензору механизма очага землетрясения, предложенному сотрудниками ИФЗ РАН [Riznichenko, 1985; Yunga, 1990], а сам параметр можно связать с обобщенным тензором сейсмического момента [Burridge, Knopoff, 1964] следующим соотношением:

$$
\tilde{M}_{0}=\lambda \tilde{s}^{0}+2 \mu \tilde{s}^{\prime}
$$

где $\lambda, \mu$ - параметры Ламе, $s^{\prime}{ }_{i k}=1 / 2 s\left(v_{i} l_{k}+l_{i} v_{k}\right)$ и $s_{i k}{ }^{0}=s v_{i} v_{k}$ - структурный параметр для локальных сдвигов и трещин соответственно. Для произвольно 


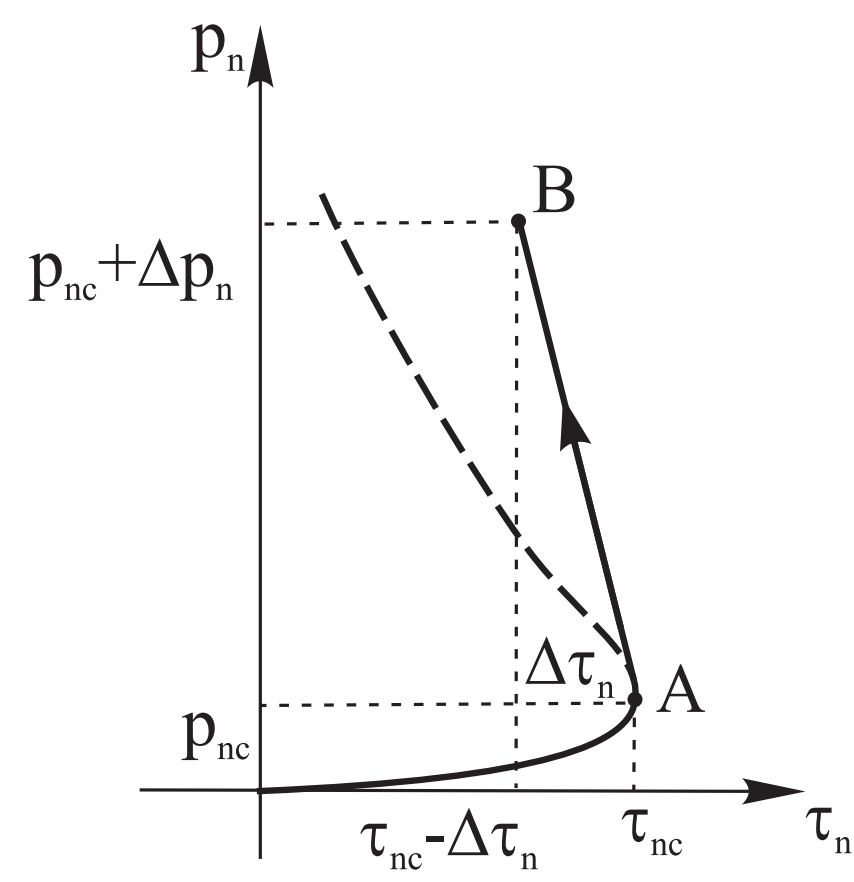

Рис. 4. Траектория эволюции геосреды с дефектами при реализации землетрясения ( $\tau_{n c}-$ критическая величина касательных напряжений, $\Delta \tau_{n}$ - величина сбрасываемых напряжений, $p_{n c}+\Delta p_{n}-$ деформация, индуцированная дефектами, определяемая по величине подвижки в очаге).

Fig. 4. The curve showing the evolution of the geomedium containing defects in case of earthquake occurrence $\left(\tau_{n c}-\right.$ critical value of tangential stresses, $\Delta \tau_{n}$ - value of released stresses, $p_{n c}+\Delta p_{n}-$ deformation induced by defects, which is determined from the value of displacement in the earthquake focus).

выбранного землетрясения, для которого современный уровень инструментальных наблюдений позволяет определить все компоненты тензора сейсмического момента, можно восстановить все компоненты тензорного структурного параметра $s_{i k}$. Это землетрясение является актом хрупкого разрушения на масштабе $L_{a}$, соизмеримо с размером очага, а на масштабе $L_{a-1}$ с линейными размерами на порядок меньше - следствием развития дефектов геосреды в режиме с обострением - результатом неустойчивого лавинообразного роста деформации, индуцированной дефектами $p_{i k}$.

Компоненты тензора деформации $p_{i k}$ на масштабе $L_{a-1}$ определяются через структурный параметр $s_{i k}$ на масштабе $L_{a}$ как

$$
\tilde{p}=\tilde{s} / S R,
$$

где $S$ - площадь очага землетрясения, $R$ - размер очага землетрясения. На диаграмме зависимости касательных напряжений $\tau_{n}$ и соответствующих им де- формаций $p_{n}$ процесс развития землетрясения представляет собой переход системы из состояния $A$ в состояние $B$ (рис. 4). Этот переход сопровождается падением напряжения $\Delta \tau_{n}$ и резким скачком индуцированной дефектами деформации до уровня $p_{n c}+\Delta p_{n}$ (определяемого соотношением (18)). Последние достижения в области реконструкции величин тектонических напряжений [Rebetskiy, 2009b; Hardebeck, Hauksson, 2001] позволяют помимо величины сбрасываемых напряжений $\Delta \tau_{n}$ определить абсолютную величину напряжения до землетрясения $\tau_{n c}$, что, в совокупности с $p_{n c}+\Delta p_{n}$, дает возможность установить критическое положение геосреды $\left(p_{n c}, \sigma_{n c}\right)$, характеризующее переход к началу формирования хрупкого разрушения.

Исследование распределения критических величин $\left(p_{n c}, \sigma_{n c}\right)$ в сейсмоактивных районах в совокупности с картами плотности сейсмогенных разрывов [Zavyalov, 1986] (фактически отражающих изменение параметра структурного скейлинга $\delta$ ) позволит продвинуться в понимании механизмов формирования землетрясений различного ранга и сформулировать новые прогностические признаки.

\section{4. ЗАКЛЮЧЕНИЕ}

Развиваемый в настоящей работе подход основан на результатах статистического описания, установивших аналогию в поведении ансамбля дефектов в поле внешнего напряжения с явлениями в неравновесных открытых системах. Введение нового параметра порядка - деформации, обусловленной дефектами, - позволило учесть суммарный вклад дефектов в деформацию среды на следующем масштабном уровне и определить возможные нелинейные пространственно-временные сценарии развития этой деформации, реализация каждого из которых зависит от второго параметра порядка - параметра структурного скейлинга. Эволюция параметра структурного скейлинга, имеющего структуру, аналогичную концентрационному критерию, определяет возможные механизмы релаксации напряжений в массиве горных пород: в виде квазипластического деформирования среды, локализованного в регулярно расположенных пространственных областях; в виде локализованной катакластической деформации, мигрирующей по пространству со скоростью, на порядки меньшей скорости звука; в виде лавиннонеустойчивого роста дефектов в локализованной пространственной области, уменьшающейся с течением времени, - очаге хрупкого разрушения. Переход к стадии катастрофического роста дефектов имеет резонансную природу и определяется слабыми флуктуациями поля напряжения, когда сформированное автомодельное распределение дефектов геосреды находится в метастабильном состоянии. Реализация режима с 
обострением в развитии дефектов геосреды характеризует появление очага хрупкого разрушения, соизмеримого с масштабом наблюдения, которое сопровождается «замораживанием» процессов деформирования в окружающих его объемах.

Математическая модель подготовки потенциального очага землетрясения как результата эволюции дефектов геосреды на различных пространственных масштабах соответствует, по мнению авторов, требованиям «новой» парадигмы и является формализованным обобщением ЛНТ-модели - моделью подготовки зем- летрясения по механизму деструкции, согласно классификации И.П. Добровольского [Dobrovol'sky, 2009].

Работа выполнена при финансовой поддержке Программы Президиума РАН «Фундаментальные проблемы механики взаимодействий в технических и природных системах» № 012-П-1-1018, Российского фонда фундаментальных исследований (гранты № 11-0596005-р_урал_а, № 12-05-00670-а) и гранта Президента РФ по государственной поддержке молодых Российских ученых-кандидатов наук (МК-6741.2013.1).

\section{5. ЛИТЕРАТУРА}

Barenblatt G.I., Botvina L.R., 1982. A note concerning power-type constitutive equations of deformation and fracture of solids. International Journal of Engineering Sciences 20 (2), 187-191. http://dx.doi.org/10.1016/0020-7225(82)90015-5.

Botvina L.R., Barenblatt G.I., 1985. Auto-modelling of damageability accumulation. Problemy Prochnosti (12), 17-24 (in Russian) [Ботвина Л.Р., Баренблатт Г.И. Автомодельность накопления повреждаемости // Проблемы прочности. 1985. № 12. C. 17-24].

Botvina L.R., Rotvain I.M., Keilis-Borok V.I., Oparina I.B., 1995. On the nature of the Gutenberg-Richter dependence in various stages of damage accumulation and earthquake preparation. Doklady AN 345 (6), 809-812 (in Russian) [Ботвина Л.Р., Ротвайн И.М., Кейлис-Борок В.И., Опарина И.Б. О характере графика повторяемости на различных стадиях дефектообразования и подготовки землетрясения // Доклады АН. 1995. Т. 345. № 6. С. 809-812].

Bowman D.D., Ouillon G., Sammis C.G., Sornette A., Sornette D., 1998. An observation test of the critical earthquake concept. Journal of Geophysical Research: Solid Earth 103 (B10), 24359-24372. http://dx.doi.org/10.1029/98JB00792.

Burridge R., Knopoff L., 1964. Body force equivalents for seismic dislocations. Bulletin of the Seismological Society of America 54 (6A), 1875-1888.

Dobrovol'sky I.P., 2009. The Mathematical Theory of Preparation and Prediction of Tectonic Earthquakes. FIZMATLIT, Moscow, 240 p. (in Russian) [Добровольский И.П. Математическая теория подготовки и прогноза тектонического землетрясения. М.: ФИЗМАТЛИТ, 2009. 240 с.].

Fedotov S.A., 2005. Long-Term Earthquake Prediction for the Kuril-Kamchatka Arc. Nauka, Moscow, 301 p. (in Russian) [Федотов С.А. Долгосрочный сейсмический прогноз для Курило-Камчатской дуги. М.: Наука, 2005. 301 с.].

Geilikman M.B., Pisarenko V.F., 1989. On self-similarity in geophysical phenomena. In: Sadovsky M.A. (Ed.), Discrete properties of the geophysical medium. Moscow, Nauka, p. 109-131 (in Russian) [Гейликман М.Б., Писаренко В.Ф. О самоподобии в геофизических явлениях // Дискретные свойства геофизической среды / Под ред. М.А. Садовского. М.: Наука, 1989. С. 109-131].

Goldin S.V., 2002. Destruction of the lithosphere and physical mesomechanics. Fizicheskaya Mezomekhanika 5 (5), 5-22 (in Russian) [Гольдин С.В. Деструкция литосферы и физическая мезомеханика // Физическая мезомеханика. 2002. T. 5. № 5. C. 5-22].

Goldin S.V., 2004. Dilatancy, repacking, and earthquakes. Izvestiya, Physics of the Solid Earth 40 (10), 817-832.

Goldin S.V., Yushin V.I., Ruzhich V.V., Smekalin O.P., 2002. Slow motions - a myth or reality. The physical basis for rock failure forecasting: Proceedings of the 9th International Workshop. Krasnoyarsk, p. 213-220 (in Russian) [Гольдин С.В., Юшин В.И., Ружич В.В., Смекалин О.П. Медленные движения - миф или реальность. Физические основы прогнозирования разрушения горных пород: Материалы 9-й международной школы-семинара. Красноярск, 2002. C. 213-220].

Gzovsky M.V., 1975. Fundamentals of Tectonophysics. Nauka, Moscow, 536 p. (in Russian) [Гзовский М.В. Основы тектонофизики. М.: Наука, 1975. 536 с.].

Hardebeck J.L., Hauksson E., 2001. Crustal stress field in Southern California and its implications for fault mechanics. Journal of Geophysical Research: Solid Earth 106 (B10), 21859-21882. http://dx.doi.org/10.1029/2001JB000292.

Hirata T., 1989. A correlation between the b value and fractal dimension of earthquakes. Journal of Geophysical Research: Solid Earth 94 (B6), 7507-7514. http://dx.doi.org/10.1029/JB094iB06p07507.

Hirata T., Satoh T., Ito K., 1987. Fractal structure of spatial distribution of microfracturing in rock. Geophysical Journal of the Royal Astronomical Society 90 (2), 369-374. http://dx.doi.org/10.1111/j.1365-246X.1987.tb00732.x.

Knopoff L., 1993. Self-organization and the development of pattern: implications for earthquake prediction. Proceedings of the American Philosophical Society 137, 339-349.

Kossobokov V.G., Keilis-Borok V.I., Turcotte D.L., Malamud B.D., 2000. Implications of a statistical physics approach to earthquake hazard assessment and forecasting. Pure and Applied Geophysics 157 (11-12), 2323-2349. http://dx.doi.org/ 


\subsection{7/PL00001086.}

Kuksenko V.S., 1986. The model of transition from micro- to macro-fracturing in solids: Proceedings of the $1^{\text {st }}$ All-Union Workshop «Physics of Strength and Plasticity». Nauka, Leningrad, p. 36-41 (in Russian) [Куксенко В.С. Модель перехода от микро- к макро-разрушению твердых тел // Сборник докладов I Всесоюз. шк.-семинара «Физика прочности и пластичности». Л.: Наука, 1986. С. 36-41].

Kurdyumov S.P., 2006. Regimes with Peaking. The Evolution of the Idea. In: G.G. Malinetsky (Ed.), 2nd Edition. Moscow, FIZMATLIT, 312 p. (in Russian) [Курдюмов С.П. Режимы с обострением. Эволюция идеи / Под ред. Г.Г. Малинецкого. М.: ФИЗМАТЛИТ, 2006. 2-е изд. 312 с.].

Kurdyumov S.P., Malinetsky G.G., 1983. Synergetics - the Theory of Self-organization. Ideas, Methods, and Prospects. Znanie, Moscow, 64 p. (in Russian) [Курдюмов С.П., Малинецкий Г.Г. Синергетика - теория самоорганизации. Идеи, методы, перспективы. М.: Знание, 1983. 64 с.].

Leonov M.G., 2011. Concentrated deformation zones and intraplate divisibility of the crust and lithosphere: Abstracts of the 5th International Symposium «Modern Problems of Geodynamics and Geoecology of Intracontinental Orogens». Bishkek, June 19-24, 2011, p. 214 (in Russian) [Леонов М.Г. Зоны концентрированной деформации и внутриплитная делимость земной коры и литосферы // Тезисы докладов. Пятый Международный симпозиум «Современные проблемы геодинамики и геоэкологии внутриконтинентальных орогенов», Бишкек, 19-24 июня 2011. С. 214].

Lockner D.A., Byerlee J.D., Kuksenko V.S., Ponomarev A., Sidorin A., 1992. Observations of quasistatic fault growth from acoustic emissions. In: B. Evans, T.-F. Wong (Eds.), Fault mechanics and transport properties of rocks. Academic Press, p. 3-31.

Lyakhovsky V.A., Ben-Zion Y., Agnon A., 1997. Distributed damage, faulting, and friction. Journal of Geophysical Research: Solid Earth 102 (B12), 27635-27649. http://dx.doi.org/10.1029/97JB01896.

Lyakhovsky V.A., Myasnikov V.P., 1985. On behavior of viscoelastic cracked solid. Izvestiya, Physics of the Solid Earth 4, 28-35.

Ma Sh., 1980. The Modern Theory of Critical Phenomena. Mir, Moscow, 295 p. (in Russian) [Ма Ш. Современная теория критических явлений. М.: Мир, 1980. 295 с.].

Makarov P.V., 2005. The loadable material as a nonlinear dynamical system. The problem of modeling. Fizicheskaya Mezomekhanika 8 (6), 39-56 (in Russian) [Макаров П.В. Нагружаемый материал как нелинейная динамическая система. Проблема моделирования // Физическая мезомеханика. 2005. Т. 8. № 6. С. 39-56].

Makarov P.V., 2008. Mathematical theory of evolution of loaded solids and media. Physical Mesomechanics 11 (5-6), 213227. http://dx.doi.org/10.1016/j.physme.2008.11.002.

Makarov P.V., 2011. The phenomenon of self-organized criticality in the evolution of the VAT and the destruction of mountains // Proceedings of the 2nd Youth Workshop "Modern Tectonophysics. Methods and Results”, Moscow, October 1721, 2011, p. 60-77 (in Russian) [Макаров П.В. Явление самоорганизованной критичности в эволюции НДС и разрушение горных массивов // Материалы Второй молодежной школы-семинара «Современная тектонофизика. Методы и результаты», Москва, 17-21 октября, 2011. С. 60-77].

Makarov P.V., Smolin I.Yu., Stefanov Yu.P., Kuznetsov P.V., Trubitsyn A.A., Trubitsyna N.V., Voroshilov S.P., Voroshilov Ya.S., 2007. The Nonlinear Mechanics of Geomaterials and Geoenvironments. Publishing House of SB RAS, GEO Branch, Novosibirsk, 235 р. (in Russian) [Макаров П.В., Смолин И.Ю., Стефанов Ю.П., Кузнецов П.В., Трубицын А.А., Трубицына Н.В., Ворошилов С.П., Ворошилов Я.С. Нелинейная механика геоматериалов и геосред. Новосибирск: Издательство СО РАН. Филиал «ГЕО», 2007. 235 с.].

Myachkin V.I., 1978. Earthquake Preparation Processes. Nauka, Moscow, 232 p. (in Russian) [Мячкин В.И. Процессы подготовки землетрясения. М.: Наука, 1978. 232 с.].

Myachkin V.I., Kostrov B.V., Shamina O.G., Sobolev G.A., 1975. Fundamentals of physics of earthquake focus and precursors. In: The physics of earthquake focus. Nauka, Moscow, p. 9-41 (in Russian) [Мячкин В.И., Костров Б.В., Шамина О.Г., Соболев Г.А. Основы физики очага и предвестники землетрясений // Физика очага землетрясения. М.: Наука, 1975. С. 9-41].

Naimark O.B., 1982. On deformation properties and the fracturing kinetics of solids with microfractures. In: On the thermodynamics of deformation and fracturing of solids with microfractures. Sverdlovsk, p. 3-34 (in Russian) [Наймарк О.Б. O деформационных свойствах и кинетике разрушения твердых тел с микротрещинами // О термодинамике деформирования и разрушения твердых тел с микротрещинами. Свердловск, 1982. С. 3-34].

Naimark O.B., 1998. Defect-induced instabilities in condensed media. Journal of Experimental and Theoretical Physics Letters 67 (9), 751-758. http://dx.doi.org/10.1134/1.567742.

Naimark O.B., 2003. Collective properties of defects ensemble and some nonlinear problems of plasticity and failure. Physical Mesomechanics 4 (4), 45-72.

Naimark O.B., 2004. Defect induced transitions as mechanisms of plasticity and failure in multifield continua. In: G. Capriz, P. Mariano (Eds.), Advances in multifield theories of continua with substructure. Boston: Birkhauser, p. 75-114.

Naimark. O.B., 2006. Structural-scaling transition in mesodefect ensembles as mechanism of relaxation and failure in shocked and dynamically loaded materials (experimental and theoretical study). Journal de Physique IV 134 (1), 3-8. http://dx.doi.org/10.1051/jp4:2006134002. 
Naimark O.B., 2008. Structural-scaling transitions and self-similar features of earthquake development. Physical Mesomechanics 11 (3-4), 187-201. http://dx.doi.org/10.1016/j.physme.2008.07.008.

Naimark O.B., Barannikov V.A., Davydova M.M., Plekhov O.A., Uvarov S.V., 2000. Crack propagation: dynamic stochasticity and scaling. Technical Physics Letters 26 (3), 254-258. http://dx.doi.org/10.1134/1.1262809.

Naimark O.B., Davydova M.M., 1996. Crack initiation and crack growth as the problem of localized instability in microcrack ensemble. Journal de Physique III 6 (C6), 259-267. http://dx.doi.org/10.1051/jp4:1996625.

Panin V.E., 1998. Fundamentals of Physical Mesomechanics. Fizicheskaya Mezomekhanika 1 (1), 5-22 (in Russian) [Панин B.E. Основы физической мезомеханики // Физическая мезомеханика. 1998. Т. 1. № 1. С. 5-22].

Panin V.E., Grinyaev Yu.V., Psakhie S.G., 2004. Physical mesomechanics: achievements over two decades of development, problems and prospects. Fizicheskaya Mezomekhanika 7 (S1-1), p. 25-40 (in Russian) [Панин В.Е., Гриняев Ю.В., Псахье С.Г. Физическая мезомеханика: достижения за два десятилетия развития, проблемы и перспективы // Физическая мезомеханика. 2004. Т. 7. № S1-1. С. 25-40].

Panteleev I.A., Plekhov O.A., Naymark O.B., 2011. Self-similarity mechanisms of damage growth in solids experiencing quasi-brittle fracture. Computational Continuum Mechanics 4 (1), 90-100. http://dx.doi.org/10.7242/1999-6691/2011.4.1.8.

Panteleev I.A., Plekhov O.A., Naimark O.B., 2012. Nonlinear dynamics of the blow-up structures in the ensembles of defects as a mechanism of formation of earthquake sources. Izvestiya, Physics of the Solid Earth 48 (6), 504-515. http://dx.doi. org/10.1134/S1069351312060055.

Rebetskii Yu.L., 2003. Development of the method of cataclastic analysis of shear fractures for tectonic stress estimation. Doklady Earth Sciences 388 (1), 72-76.

Rebetskii Yu.L., 2007a. New data on natural strains within the area of preparation for strong earthquake. The model of the earthquake source. Geophysical Journal 29 (6), 96-115 (in Russian) [Ребецкий Ю.Л. Новые данные о природных напряжениях в области подготовки сильного землетрясения. Модель очага землетрясения // Геофизический журнал. 2007. Т. 29. № 6. С. 96-115].

Rebetskii Yu.L., 2007b. Condition and problems of theories of earthquakes prediction. Analysis of bases from position of appointed approach. Geophysical Journal 29 (4), 92-110 (in Russian) [Ребецкий Ю.Л. Состояние и проблемы теорий прогноза землетрясений. Анализ основ с позиции детерминированного подхода // Геофизический журнал. 2007. T. 29. № 4. С. 92-110].

Rebetskii Yu.L., 2009a. The third and the fourth stages of strains reconstruction in the method of cataclastic analysis of shift ruptures. Geophysical Journal 31 (2), 93-106 (in Russian) [Ребецкцй Ю.Л. Третий и четвертый этапы реконструкции напряжений в методе катакластического анализа сдвиговых разрывов // Геофизический журнал. 2009. Т. 31. № 2. C. 93-106].

Rebetskii Yu.L., 2009b. Estimation of stress values in the method of cataclastic analysis of shear fractures. Doklady Earth Sciences 428 (7), 1202-1207. http://dx.doi.org/10.1134/S1028334X09070368.

Riznichenko Yu.V., 1985. Problems of Seismology. Selected Works. Nauka, Moscow, 408 p. (in Russian) [Ризниченко Ю.В. Проблемы сейсмологии. Избранные труды. М.: Наука, 1985. 408 с.].

Rundle J.B., 1988. A physical model for earthquakes, 1. Fluctuation and interactions. Journal of Geophysical Research: Solid Earth 93 (B6), 6237-6254. http://dx.doi.org/10.1029/JB093iB06p06237.

Rundle J.B., 1989. A physical model for earthquakes, 3. Thermodynamic approach and its relation to nonclassical theories of nucleation. Journal of Geophysical Research: Solid Earth 94 (B3), 2839-2855. http://dx.doi.org/10.1029/JB094iB03 p02839.

Rundle J.B., Gross S., Klein W, Ferguson C., Turscotte D.L., 1997. The statistical mechanics of earthquakes. Tectonophysics 277 (1-3), 147-164. http://dx.doi.org/10.1016/S0040-1951(97)00083-8.

Rundle J.B., Klein W., Gross S., 1999. Physical basis for statistical patterns in complex earthquake populations: models, predictionsand tests. Pure and Applied Geophysics 155 (2-4), 575-607. http://dx.doi.org/10.1007/s000240050278.

Sadovsky M.A., 1989. Discrete Properties of Geophysical Medium. Nauka, Moscow, 174 p. (in Russian) [Caдовский M.A. Дискретные свойства геофизической среды. М.: Наука, 1989. 174 с.].

Sadovsky M.A., Bolkhovitinov L.G., Pisarenko V.F., 1987. Deformation of Geophysical Medium and Seismic Process. Nauka, Moscow, 100 p. (in Russian) [Садовский М.А., Болховитинов Л.Г., Писаренко В.Ф. Деформирование геофизической среды и сейсмический процесс. М.: Наука, 1987. 100 с.].

Sadovsky M.A., Pisarenko V.F., 1989. Randomness and instability in geophysical processes // Izvestiya AN SSSR. Fizika Zemli (2), 3-12 (in Russian) [Садовский M.A., Писаренко В.Ф. Случайность и неустойчивость в геофизических процессах // Известия АН СССР. Физика Земли. 1989. № 2. С. 3-12].

Seminsky K.Zh., 2009. Tectonophysical analysis of the internal structure of fault zones. Modern tectonophysics. Methods and results // Proceedings of the 1st Youth Workshop, Moscow, p. 258-276 (in Russian) [Семинский К.Ж. Тектонофизический анализ внутренней структуры разломных зон // Современная тектонофизика. Методы и результаты. Материалы первой молодежной школы-семинара, Москва, 2009. С. 258-276].

Sherman S.I., 1977. Physical Regularities of Development of Crustal Faults. Nauka, Novosibirsk, 102 p. (in Russian) [Шерман С.И. Физические закономерности развития разломов земной коры. Новосибирск: Наука, 1977.102 с.].

Sherman S.I., Seminsky K.Zh., 2010. Tectonophysical research at the Institute of the Earth's crust SB RAS: major achieve- 
ments and actual problems. Geodynamics \& Tectonophysics 1 (1), 4-23. http://dx.doi.org/10.5800/GT-2010-1-1-0003.

Sherman S.I., Seminsky K.Zh., Bornyakov S.A., Adamovich A.N., Gladkov A.S., 2000. Theoretical and practical implications of the development of M.V. Gzovsky's ideas in researches of the Institute of Earth's crust // M.V. Gzovsky and development of tectonophysics. Chief editors Yu.G. Leonov, V.N. Strakhov. Nauka, Moscow, p. 245-265 (in Russian) [Шерман С.И., Семинский К.Ж., Борняков С.А., Адамович А.Н., Гладков А.С. Теоретические и практические следствия развития идей М.В. Гзовского в исследованиях Института земной коры СО РАН // М.В. Гзовский и развитие тектонофизики / Отв. ред. Ю.Г. Леонов, В.Н. Страхов. М.: Наука, 2000. С. 245-265].

Sherman S.I., Seminsky K.Zh., Cheremnykh A.V., 1999. Destructive zones and fault-block structures of the Central Asia. Tikhookeanskaya Geologiya 18 (2), 41-53 (in Russian) [Шерман С.И., Семинский К.Ж., Черемных А.В. Деструктивные зоны и разломно-блоковые структуры Центральной Азии // Тихоокеанская геология. 1999. Т. 18. № 2. С. 41-53].

Sobolev G.A., Ponomarev A.V., 2003. Physics of Earthquakes and Precursors. Nauka, Moscow, 270 p. (in Russian) [Соболев Г.А., Пономарев А.В. Физика землетрясений и предвестники. М.: Наука, 2003. 270 с.].

Sobolev G.A., Tyupkin Yu.S., 2000. Analysis of energy release process during main rupture formation in laboratory studies of rock fracture and before strong earthquakes. Izvestiya, Physics of the Solid Earth 36 (2), 138-149.

Sornette D., 2000. Critical phenomena in natural sciences: Chaos, Fractals, Self-Organization and Disorder: Concepts and Tools (Springer Series in Synergetics). Springer-Verlag, Heidelberg, 423 p.

Strakhov V.N., 1989. Towards a new paradigm of seismology. Priroda (12), 4-9 (in Russian) [Страхов B.Н. К новой парадигме сейсмологии // Природа. 1989. № 12. С. 4-9].

Tyupkin Yu.S., 2004a. Dynamics of the formation of a potential earthquake source. Izvestiya, Physics of the Solid Earth 40 (3), 198-205.

Tyupkin Yu.S., 2004b. Potential earthquake source: generalization to the theory with spatial derivative. Izvestiya, Physics of the Solid Earth 40 (10), 833-839.

Tyupkin Yu.S., 2004c. Formation of potential earthquake focus: an analogy with phase transition. Vychislitelnaya Seismologiya 35, 296-311 (in Russian) [Тюпкин Ю.С. Формирование потенциального очага землетрясения: аналогия с фазовым переходом // Вычислительная сейсмология. 2004. Вып. 35. С. 296-311].

Tyupkin Yu.S., 2007. Earthquake source nucleation as self organization process. Tectonophysics 431 (1-4), 73-81. http://dx. doi.org/10.1016/j.tecto.2006.04.022.

Tyupkin Yu.S., di Giovambattista R., 2005. Correlation length as an indicator of critical point behavior prior to a large earthquake. Earth and Planetary Science Letters 230 (1-2), 85-96. http://dx.doi.org/10.1016/j.epsl.2004.10.037.

Varnes D.J., 1989. Predicting earthquakes by analyzing accelerating precursory seismic activity. Pure and Applied Geophysics 130 (4), 661-686.

Yunga S.L., 1990. Methods and Results of Studies of Seismotectonic Deformations. Nauka, Moscow, 191 p. (in Russian) [Юнга С.Л. Методы и результаты изучения сейсмотектонических деформаций. М.: Наука, 1990. 191 с.].

Zaliapin V., Keilis-Borok V.I., Ghil M., 2003. A Boolean delay model of colliding cascades, prediction of critical transitions. Journal of Statistical Physics 111 (3-4), 839-861. http://dx.doi.org/10.1023/A:1022802432590.

Zavyalov A.D., 1986. The parameter of concentration of seismogenic faults as a precursor of strong earthquakes in Kamchatka. Vulkanalogiya i Seimologiya (3), 58-71 (in Russian) [Завьялов А.Д. Параметр коцентрации сейсмогенных разрывов как предвестник сильных землетрясений Камчатки // Вулканология и сейсмология. 1986. № 3. С. 5871].

Zhurkov S.N.,1968. The kinetic concept of the strength of solids. Vestnik AN SSSR (3), 46-52 (in Russian) [Журков C.H. Кинетическая концепция прочности твердых тел // Вестник АН СССР. 1968. Вып. 3. С. 46-52].

Zöller G., Hainzl S., Kurths J., 2001. Observation of growing correlation length as an indicator for critical point behavior prior to large earthquakes. Journal of Geophysical Research: Solid Earth 106 (B2), 2167-2176. http://dx.doi.org/10. 1029/2000JB900379.

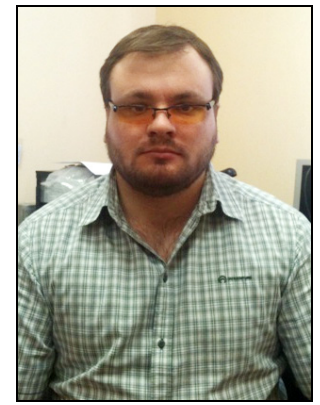

Пантелеев Иван Алексеевич, канд. физ.-мат. наук, м.н.с.

Институт механики сплошных сред УрО РАН

614013, Пермь, ул. ак. Королева, 1, Россия

Тел.: (342) 2378312; $₫$ e-mail: pia@icmm.ru

Panteleev, Ivan A., Candidate of Physics and Mathematics, Junior Researcher Institute of Continuous Media Mechanics, Ural Branch of RAS, Perm, Russia 1 Academician Korolev street, Perm 614013, Russia

Tel.: (342) 2378312; \ e-mail: pia@icmm.ru 


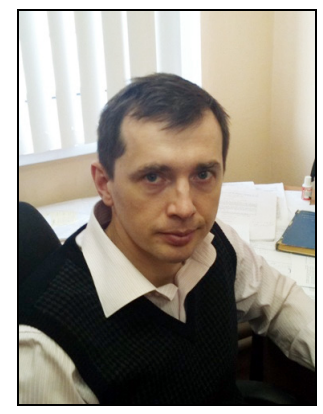

Плехов Олег Анатольевич, докт. физ.-мат. наук, с.н.с.

Институт механики сплошных сред УрО РАН

614013, Пермь, ул. ак. Королева, 1, Россия

Тел.: (342) 2378312; e-mail: poa@icmm.ru

Plekhov, Oleg A., Doctor of Physics and Mathematics, Senior Researcher Institute of Continuous Media Mechanics, Ural Branch of RAS, Perm, Russia

1 Academician Korolev street, Perm 614013, Russia

Tel.: (342) 2378312; e-mail: poa@icmm.ru

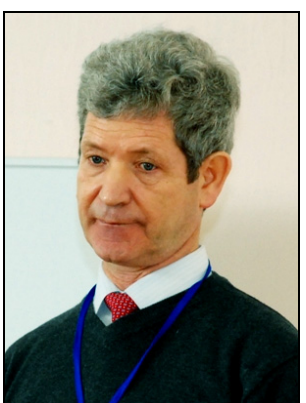

Наймарк Олег Борисович, докт. физ.-мат. наук, заведующий лабораторией Институт механики сплошных сред УрО РАН

614013, Пермь, ул. ак. Королева, 1, Россия

Тел.: (342) 2378312; e-mail: naimark@icmm.ru

Naimark, Oleg B., Doctor of Physics and Mathematics, Head of Laboratory Institute of Continuous Media Mechanics, Ural Branch of RAS, Perm, Russia

1 Academician Korolev street, Perm 614013, Russia

Tel.: (342) 2378312; e-mail: naimark@icmm.ru 